\title{
Ivabradine reduces baseline and stress-induced increase of heart rate and blood pressure and modulates neuroendocrine stress response in rats depending on stressor intensity
}

\author{
Katarina Ondicova $^{1 \star}$, Noemi Hegedusova ${ }^{1 \star}$, Miroslav Tibensky ${ }^{2,3}$ and Boris Mravec ${ }^{2,3}$ \\ ${ }^{1}$ Institute of Pathophysiology, Faculty of Medicine, Comenius University in Bratislava, Bratislava, Slovakia \\ ${ }^{2}$ Institute of Physiology, Faculty of Medicine, Comenius University in Bratislava, Bratislava, Slovakia \\ ${ }^{3}$ Institute of Experimental Endocrinology, Biomedical Research Center, Slovak Academy of Sciences, Bratislava, Slovakia
}

\begin{abstract}
Ivabradine, a selective inhibitor of the sinoatrial pacemaker, is used in clinical practice to reduce heart rate. However, its potential effect on the neuroendocrine stress response has not been investigated. Therefore, we determined the effect of administering ivabradine to rats on cardiovascular parameters and plasma levels of epinephrine, norepinephrine, and corticosterone. Ivabradine was administered intraperitoneally 30 min before exposing animals to either handling, restraint, or immobilization stress. Heart rate and blood pressure were monitored telemetrically. Blood samples were collected before, during, and after stressor exposure to determine the extent of the neuroendocrine stress response as reflected by plasma epinephrine, norepinephrine, and corticosterone levels. In animals pretreated with ivabradine, significantly lower values of heart rate and blood pressure were found during both the baseline period and during exposure to stressors, as well as during the rest period following stressor exposure. Ivabradine also significantly reduced handling-induced epinephrine and norepinephrine release into the bloodstream. However, ivabradine significantly potentiated restraint- and immobilization-induced increases of plasma epinephrine levels, whereas stress-induced changes in plasma norepinephrine and corticosterone levels were ambiguous. Our data shows that ivabradine significantly reduces blood pressure in rats during both baseline and stressful conditions, and also affects the neuroendocrine stress response. These findings show that viscerosensory signaling from the cardiovascular system may significantly modulate the neuroendocrine stress response.
\end{abstract}

Key words: Blood pressure - Corticosterone - Epinephrine - Heart rate - Ivabradine - Norepinephrine

\section{Introduction}

The stress response is a highly orchestrated reaction of an organism to external or internal factors (stressors) that disrupt homeostasis (Ulrich-Lai and Herman 2009). Crucial components of the neuroendocrine stress response include the sympathoadrenal system (SAS) and hypothalamo-

\footnotetext{
* These authors contributed equally to this work. Correspondence to: Boris Mravec, Institute of Physiology, Faculty of Medicine, Comenius University in Bratislava, Sasinkova 2, 81372 Bratislava, Slovakia

E-mail: boris.mravec@fmed.uniba.sk
}

pituitary-adrenocortical axis (HPA) (Delahunt and Mellsop 1987). Whereas a balanced neuroendocrine stress response is essential for survival, altered activation of the SAS or HPA axis, in either magnitude or duration, might have detrimental effects on the organism (Chrousos 2009; McEwen and Gianaros 2010; Masini et al. 2011). Therefore, the activity of the SAS and HPA axis are modulated by several mechanisms, including feedback signaling from visceral organs (Jänig 2006).

Although the effect of increased SAS activity on cardiovascular system function has been well described (Goldstein 1995), the role of signals transmitted from the cardiovascular system to the brain in modulating SAS and HPA axis activ- 
ity during stressful situations remains unclear. Increased heart rate, accompanied by more frequent activation of heart mechanoreceptors, represents a typical response to psychosocial and physical stressors (Lipp and Anderson 1999). While these changes in heart rate detected by cardiac mechanoreceptors are known to be transmitted to the brain (Peyronnet et al. 2016), it is unclear whether or not these signals affect the neuroendocrine stress response.

Several methodological issues make it difficult to investigate the role of viscerosensory signaling from the heart in modulating the neuroendocrine stress response. Firstly, it is difficult to selectively manipulate heart rate without affecting the regulatory effects of the neuroendocrine stress response on peripheral tissues and organs via the SAS and HPA axis. For example, cardiac denervation in patients after heart transplantation leads to damage of both viscerosensory and autonomic motor nerve fibers (Raczak et al. 1999; Pozza et al. 2006). Additionally, pharmacological approaches exert a low degree of selectivity. $\beta$-blockers, besides causing a reduction of heart rate, also affect the activity of other visceral organs and tissues, which induces various changes to the internal environment (Guglin 2013) that are subsequently signaled to the brainstem and other structures involved in regulating stress-related neuroendocrine stress responses. Fortunately, ivabradine, a highly selective inhibitor of the sinoatrial funny current, was developed in the 1990's. Ivabradine selectively blocks f-channels on cardiac pacemaker cells that determine spontaneous electrical pacemaker activity in the sinoatrial node. This reduced current inhibits slow diastolic depolarization, thereby reducing heart rate (Thollon et al. 1994; Bois et al. 1996; DiFrancesco and Camm 2004). It was also found that ivabradine may affect blood flow in some vascular beds (Gardiner et al. 1995). However, there are no data showing that ivabradine has a significant effect on the activity of endocrine glands or other visceral tissues and organs. In addition, ivabradine does not cross the blood-brain barrier (Young et al. 2014) and therefore cannot directly affect brain functions. Based on these data, we propose that this drug provides an opportunity to investigate the effect of selective inhibition of heart rate on various physiological functions, including activity of the SAS and HPA axis.

Base on the above-mentioned facts, using ivabradine, a drug that selectively reduces heart rate without affecting the activity of other visceral organs and tissues in the body (DiFrancesco 2006; Nawarskas et al. 2015), we investigated the role of signaling that accompanies a stress-induced rise in heart rate on the extent of the neuroendocrine stress response. In our study, rats pretreated by ivabradine were exposed to handling, restraint stress, or immobilization while plasma epinephrine (EPI), norepinephrine (NE), and corticosterone levels were assayed. Furthermore, to confirm the efficacy of ivabradine-induced attenuation of stress-induced increases in heart rate, cardiovascular parameters were monitored telemetrically.

\section{Materials and Methods}

\section{Animals}

Male Sprague-Dawley rats weighing 250-300 g (Charles River Laboratories International Inc, Germany) were housed three per cage under controlled conditions (12/12 h light/ dark cycle with lights on at 6:00 a.m., temperature $22 \pm 1^{\circ} \mathrm{C}$ ), with food and water provided ad libitum. Experiments were performed between 08:00-12:00 a.m. The experiments were carried out in accordance with the Council Directive 2010/63EU of the European Parliament and the Council of $22^{\text {nd }}$ September 2010 on the protection of animals used for scientific purposes.

\section{Experimental design}

We performed two series of experiments. In the first experiment, the effects of ivabradine pretreatment on cardiovascular function in rats exposed to fear-inducing stressors were analyzed using telemetric devices. In the second experiment, the effects of ivabradine on neuroendocrine stress responses in rats was determined by measuring plasma EPI, NE, and corticosterone levels using immunoassay methods.

\section{Exposure of animals to stressors}

Three stressors: handling, restraint stress, and immobilization, each differing in their extent of activation of the neuroendocrine stress response were used and were performed as previously described (Kvetnansky and Mikulaj 1970; Day et al. 2005). Handling, a mild psychological stressor, was performed by gentle manipulation of the animals. To do this, each animal was removed from the cage and gently manipulated using both hands. Because animals accommodate to this stressor relatively fast, rats in our experiments were only handled for $5 \mathrm{~min}$. Restraint, a stronger stressor with predominant psychological and less intense physical components, was performed by placing the animal into a plastic tube that restricted its movements. Immobilization, a strong stressor with both physical and psychological components, was performed by taping of all four limbs to metal holders attached to an immobilization board. As a result of this the animal was not able to move, breathing was hampered, and acral parts of limbs were ischemic (Kvetnansky et al. 1971, 1978).

\section{Attenuation of stress-induced increase of heart rate by iva- bradine pretreatment}

Ivabradine (Procoralan $5 \mathrm{mg}$, Les Laboratoires Servier, Neuillysur-Seine, France) was dissolved in saline and injected intraperitoneally (i.p. $5 \mathrm{mg} / \mathrm{kg}$ b.w.) in rats (IVA group). The vehicle-treated rats were given an i.p. injection of saline (SAL 
group). Ivabradine or saline in a volume of $2 \mathrm{ml} / \mathrm{kg}$ were administered $30 \mathrm{~min}$ before exposure of animals to stressor. The dose of ivabradine was chosen based on our pilot experiment in which we have found that this dose is sufficient to prevent stress-induced increase of heart rate.

\section{Monitoring of cardiovascular system activity}

In the first experiment, rats were randomly divided into IVA and SAL groups and exposed to $5 \mathrm{~min}$ handling $(n=4), 60 \mathrm{~min}$ restraint $(n=4)$, or 60 min immobilization $(n=4)$. Heart rate $(\mathrm{HR})$ and mean arterial pressure (MAP) were recorded by telemetric devices with Millar catheters (model TRM54P, Telemetry Research, Auckland, New Zealand) implanted into the abdominal aorta seven days prior to the exposure of rats to the stressors (for details of implantation of telemetry devices see (Muntzel et al. 2012). Briefly, animals were anesthetized with an intramuscular administration of a ketamine (Narkamon 5\%; $1.2 \mathrm{ml} / \mathrm{kg}$ ) and xylazine (Rometar 2\%; $0.4 \mathrm{ml} / \mathrm{kg}$ ) mixture. The tip of the Millar catheter was placed into the aorta above the bifurcation of its abdominal part. The transmitter was placed into the abdominal cavity and attached to the peritoneum using sutures. The abdomen was then closed in two layers with interrupted sutures. To avoid post-operational infection, intramuscular injection of antibiotics (penicillin) was given immediately after implantation. After surgery, rats were housed separately with free access to tap water and pelleted food.

The recording of cardiovascular parameters started $60 \mathrm{~min}$ prior to the exposure to stressors. This interval represented baseline values analogical to the control interval used during blood sampling in the second experiment (see below). These cardiovascular parameters were continuously recorded during the entire exposure to stressors and also for the next $120 \mathrm{~min}$ (handling) or $60 \mathrm{~min}$ (restraint, immobilization) as an analogy to the rest interval during blood sampling (see below).

\section{Determination of neuroendocrine stress response}

In the second experiment, rats were randomly divided into IVA and SAL groups and exposed to handling $(n=17)$, restraint $(n=18)$, or immobilization $(n=13)$ stress, respectively. Blood samples $(0.4 \mathrm{ml})$ were collected at defined time intervals via a cannula implanted into the jugular vein one day prior to the exposure of animals to stressors. The cannulation of the jugular vein allows for repeated blood sampling without inducing any stress effects and was performed as previously described (Thrivikraman et al. 2002). Briefly, animals were anesthetized with a mixture of ketamine-xylazine as described above. The rats were then fixed in a supine position to an acrylic surgery platform. The area of incision (15 mm long) was on the right shoulder close to the base of the neck. To reach the jugular vein it was necessary to separate the surrounding muscle and membranous tissue. The exposed jugular vein was cut with spring-scissors on the upper surface and a polyethylene tube (silicon tubing, PE 50; Becton-Dickinson, Parsippany, NJ) filled with heparinized saline $(300 \mathrm{IU} / \mathrm{ml})$ was carefully placed into the right jugular vein. About $0.1 \mathrm{ml}$ of the heparinized saline was then infused through the catheter and a slow withdrawal of blood was attempted to confirm correct cannulation procedure. The cannula was tied to the vein with rostral and caudal ligatures to avoid occluding the cannula. Following this, the rat was removed from the platform and we made a small incision in the center of the rat's nape with a trochar. The end of the cannula was advanced into the trochar and exteriorized at the other end of the ventral incision, and then attached with medical thread. After cannulation, the rats were housed individually. Exposure to stressors and blood sampling started on the next day after the overnight recovery. The duration of the 1 day recovery was selected according to our previous experiments and findings showing that jugular vein cannulation does not increase baseline corticosterone levels 1 day after cannulation and does not alter subsequent stress responses in animals exposed to stressors 1 or more days after the surgical procedure (Ling and Jamali 2003).

Approximately $30 \mathrm{~min}$ before of the start of blood sampling, the free end of the jugular catheter was connected with a longer cannula to allow unstressed blood collection. Before ivabradine injection, the first blood samples (control samples) were collected from unstressed rats. Afterwards, the blood samples were collected via cannula at the time points of 0 and 5 min during handling and at 15, 30,60, and 120 min after handling (rest phase). During restraint and immobilization, blood samples were collected at the $5,15,30$, and 60 min time points during exposure, as well as $60 \mathrm{~min}$ after the end of exposure to stressor (rest phase). To replace the lost volume of blood $(0.4 \mathrm{ml})$ each rat was administered an equal volume of heparinized saline $(50 \mathrm{IU} / \mathrm{ml})$ by cannula.

\section{Biochemical analysis of plasma}

Immediately after collecting blood samples, Eppendorf vials containing the collected blood were placed on ice and centrifuged at $3000 \times g$ for $15 \mathrm{~min}$ at $4^{\circ} \mathrm{C}$ to separate plasma. Plasma samples were stored at $-70^{\circ} \mathrm{C}$ until analyzed. Plasma catecholamine concentrations were determined by a commercially available enzyme immunoassay kit (2-CAT (A-N) Research ELISA, LDN, Nordhorn, Germany). The minimum detection limit for catecholamines in this ELISA kit is $8.1 \mathrm{pg} / \mathrm{ml}$ for EPI and $5.4 \mathrm{pg} / \mathrm{ml}$ for $\mathrm{NE}$ (depending on sample volume). Plasma corticosterone concentration was determined by a commercially available radioimmunoassay kit (Corticosterone rat/mouse RIA kit, DRG Diagnostic, Germany). The minimum detection limit for corticosterone in these RIA kits is $7.7 \mathrm{ng} / \mathrm{ml}$. 


\section{Statistical analysis}

Statistical analysis was performed using GraphPad Prism 5 program (GraphPad Software, San Diego CA, USA). Statistical differences between the groups were determined by twoway analyses of variance (ANOVA) with repeated measures followed by post hoc, pair wise comparisons using Bonferroni's correction and an unpaired Student's $t$-test. Data are presented as mean \pm SEM and represent the mean for 4 rats in experiments monitoring cardiovascular activity and 8-10 rats in experiments determining the neuroendocrine stress response. The $p$ value of $<0.05$ was taken as indicative of statistical significance. The area under the curve (AUC) was calculated using the trapezoidal rule. The AUC represents an integrated value of the total amount of hormone released during the $5 \mathrm{~min}$ of handling, $60 \mathrm{~min}$ of restraint stress, or 60 min of immobilization.

\section{Results}

The effect of ivabradine on heart rate and mean arterial pressure during handling, restraint stress, or immobilization

Continual telemetric recording demonstrated that heart rate and mean arterial pressure increased only slightly after i.p. injection of saline ( $-30 \mathrm{~min}$ interval) compared to pre-injection period ( $-35 \mathrm{~min}$ interval). Furthermore, before exposure to stressors (handling, restrain or immobilization; $-5 \mathrm{~min}$

\section{Handling}
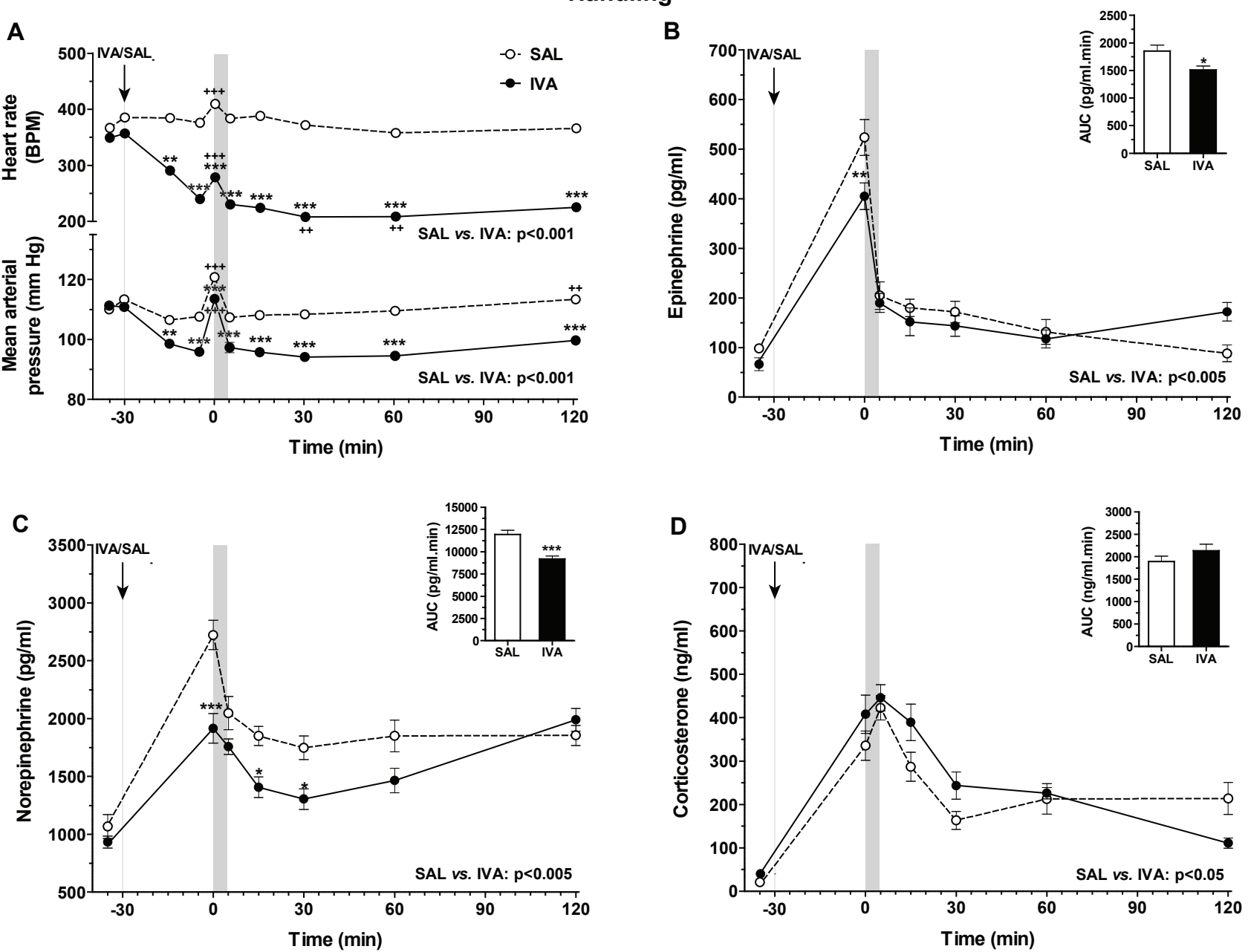

Figure 1. The effect of saline (SAL) or ivabradine (IVA) pretreatment on heart rate and mean arterial pressure (A), plasma epinephrine (B), norepinephrine (C), and corticosterone (D) levels in rats exposed to handling (grey areas). Areas under the curve (AUC) were measured from 0 to $5 \mathrm{~min}$ of handling for the plasma catecholamines or corticosterone concentrations. Each value is the mean \pm SEM ( $n=4$ for cardiovascular parameters; $n=8-9$ for hormones). Statistical significance compared to saline-pretreated group: ${ }^{\star} p<0.05 ;{ }^{\star \star} p<$ $0.01{ }^{* * *} p<0.001$; differences between corresponding pre-stress interval $(-5 \mathrm{~min})$ and following intervals: ${ }^{++} p<0.01 ;^{+++} p<0.001$. 
interval) values of these cardiovascular parameters slightly lower or even reached control values in animals injected by saline (Fig. 1A, 2A, 3A). In contrast, injection of ivabradine 30 min prior to exposure to the stressor significantly reduced values of heart rate and mean arterial pressure during the time intervals prior to the exposure of animals to stressors (-15 min and -5 min; Fig. $1 \mathrm{~A}, 2 \mathrm{~A}, 3 \mathrm{~A})$.

Whereas exposure of saline-treated rats to stressors induced increase of heart rate and blood pressure, animals injected with ivabradine showed significantly reduced values of heart rate and mean arterial pressure during exposure to stressors, as well as during following rest period (Handling HR: $\mathrm{F}_{1,60}=6496.45, p<0.001$, Handling MAP: $\mathrm{F}_{1,60}=721.53$, $p<0.001$, Fig. 1A; Restrain HR: $\mathrm{F}_{1,54}=1477.63, p<0.001$, Restrain MAP: $\mathrm{F}_{1,54}=257.58, p<0.001$; Fig. 2A; Immobilization HR: $\mathrm{F}_{1,60}=434.72, p<0.001$, Immobilization MAP: $\mathrm{F}_{1,54}=298.72, p<0.001$; Fig. 3A).

When comparing the -5 min pre-stress interval with the subsequent stress and post-stress rest intervals, exposure to stressors induces a significant increase of HR and MAP in both saline- and ivabradine-pretreated rats. This increase was more prominent in saline-treated animals than those injected with ivabradine. In handled rats this increase was found at the beginning of the handling procedure, whereas by the end of handling, HR values did not differ from the prestress $-5 \mathrm{~min}$ interval (Fig. 1A). In rats exposed to restraint,

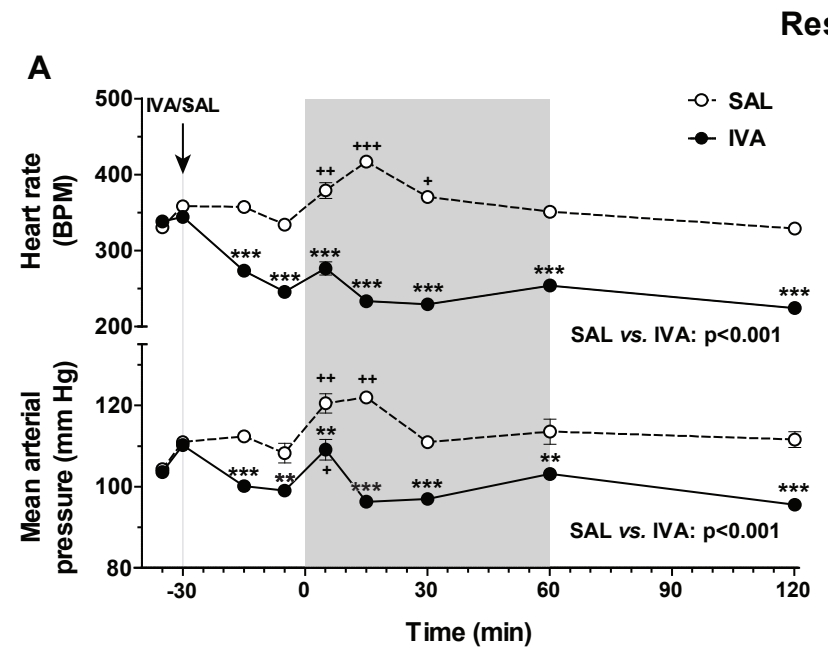

\section{Restraint}
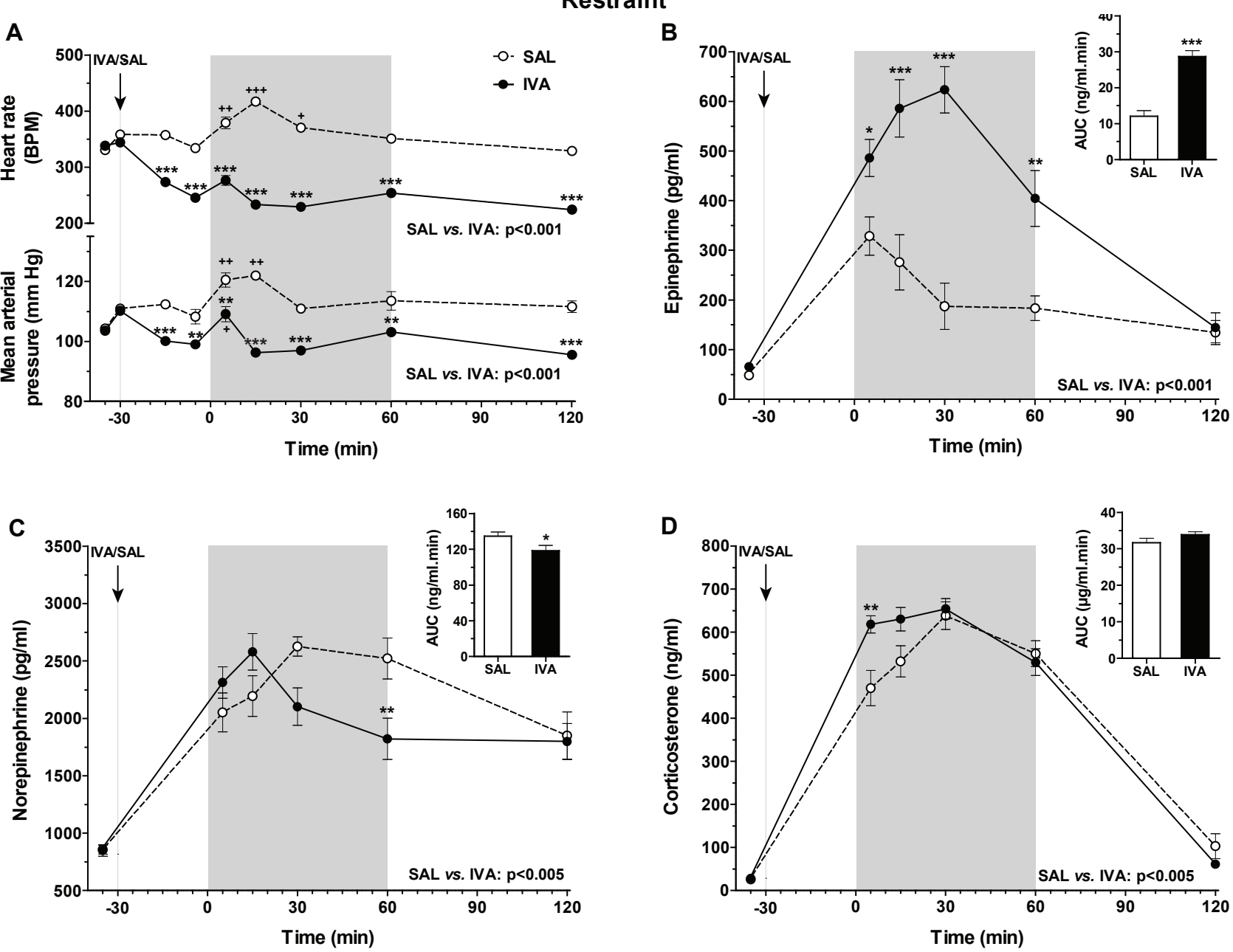

Figure 2. The effect of saline (SAL) or ivabradine (IVA) pretreatment on heart rate and mean arterial pressure (A), plasma epinephrine (B), norepinephrine (C), and corticosterone (D) levels in rats exposed to restraint stress (grey areas). Areas under the curve (AUC) were measured from 0 to $60 \mathrm{~min}$ of restraint stress for plasma catecholamines and corticosterone concentrations. Each value is the mean $\pm \operatorname{SEM}\left(n=4\right.$ for cardiovascular parameters; $n=8-10$ for hormones). Statistical significance compared to saline-pretreated group: ${ }^{\star} p<$ $0.05 ;{ }^{* *} p<0.01 ;{ }^{* * *} p<0.001$; differences between corresponding pre-stress interval $(-5 \mathrm{~min})$ and following intervals: ${ }^{+} p<0.05 ;{ }^{++} p<$ $0.01{ }^{+++} p<0.001$. 

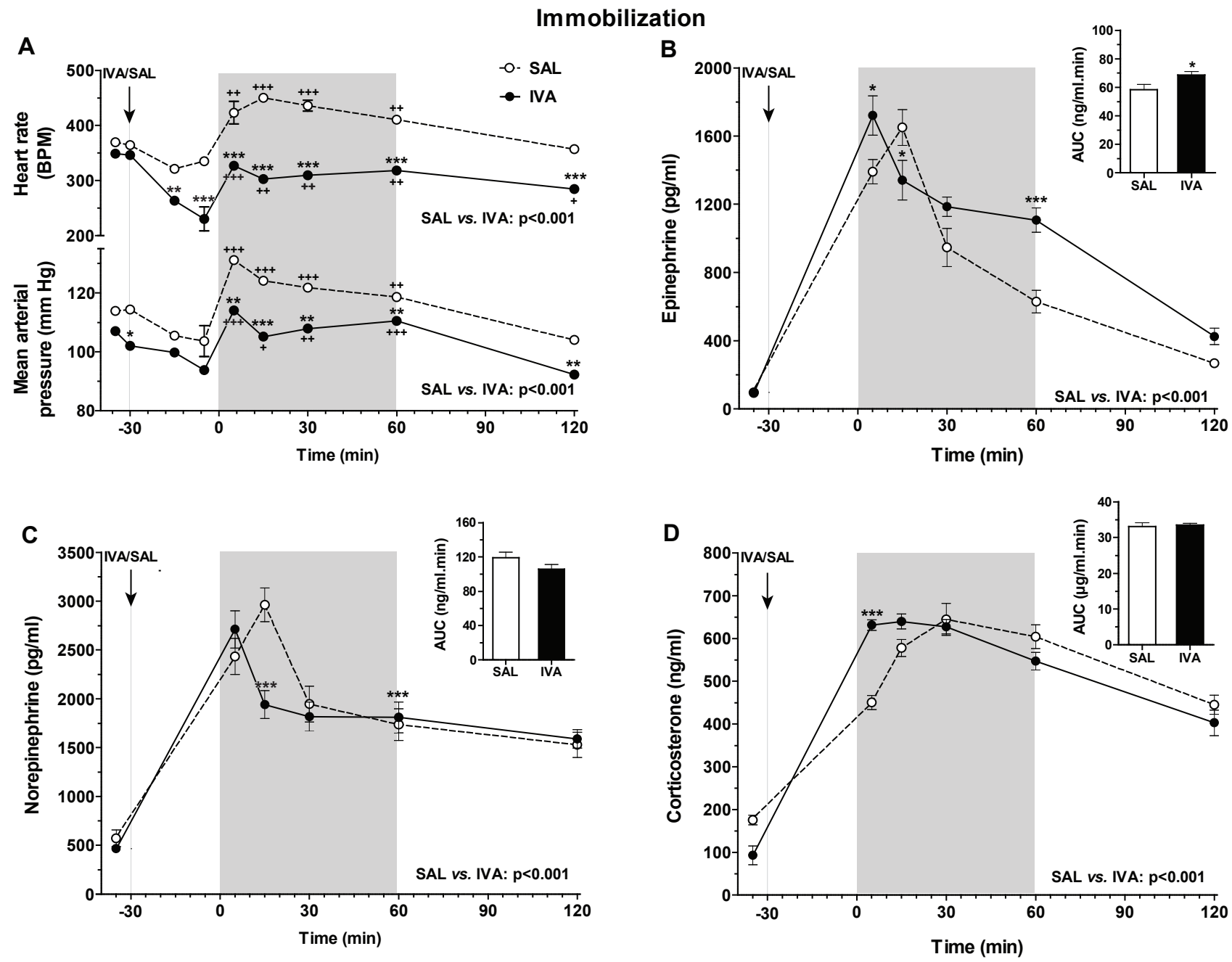

Figure 3. The effect of saline (SAL) or ivabradine (IVA) pretreatment on heart rate and mean arterial pressure (A), plasma epinephrine (B), norepinephrine (C), and corticosterone (D) levels in rats exposed to immobilization (grey areas). Areas under the curve (AUC) were measured from 0 to $60 \mathrm{~min}$ of immobilization for plasma catecholamines and corticosterone concentrations. Each value is the mean \pm SEM ( $n=4$ for cardiovascular parameters; $n=6-7$ for hormones). Statistical significance compared to the saline-pretreated group: ${ }^{\star} p<0.05 ;{ }^{* *} p<0.01 ;{ }^{* *} p<0.001$; differences between corresponding pre-stress interval ( $\left.-5 \mathrm{~min}\right)$ and following intervals: ${ }^{+} p<0.05$; ${ }^{++} p<0.01 ;{ }^{+++} p<0.001$.

increased values of the observed cardiovascular parameters were found in several stress intervals (Fig. 2A), whereas in immobilized animals HR and MAP values were increased during the entire period of immobilization (Fig. 3A).

The effect of ivabradine on plasma catecholamines and corticosterone levels during handling, restraint stress, or immobilization

In rats exposed to handling, ivabradine pretreatment significantly decreased the total amount of EPI and NE released during the $5 \mathrm{~min}$ handling period compared to the SAL group (EPI AUC: $\mathrm{t}_{16}=2.668, p<0.05$, NE AUC: $\mathrm{t}_{16}=$
5.217, $p<0.001$; Fig. 1B, C). On the other hand, ivabradine pretreatment significantly exaggerated restraint- and immobilization-induced increases of plasma EPI (Restrain EPI: $\mathrm{F}_{1,96}=70.62, p<0.001$, Fig. $2 \mathrm{~B}$; Immobilization EPI: $\mathrm{F}_{1,66}=13.94, p<0.001$, Fig. 3B). Also, the total amount of EPI released during the 60 min restraint stress was significantly higher in the IVA group compared to saline-treated animals $\left(\mathrm{t}_{16}=9.010, p<0.001\right.$; Fig. $\left.2 \mathrm{~B}\right)$, as well as during the 60 min immobilization $\left(\mathrm{t}_{11}=2.452, p<0.05\right.$; Fig. $\left.3 \mathrm{~B}\right)$. Similar to handling, the total amount of released NE was significantly decreased in ivabradine-pretreated rats compared to the SAL group during restraint stress $\left(t_{16}=2.172\right.$, $p<0.05$; Fig. 2C). In immobilized rats, the total amount 
of released NE was reduced only slightly in rats pretreated by ivabradine $\left(\mathrm{t}_{16}=1.488, p=0.1488\right.$, SAL: $119 \pm 7 \mathrm{ng} / \mathrm{ml} /$ min vs. IVA: $106 \pm 5 \mathrm{ng} / \mathrm{ml} / \mathrm{min}$; Fig. 3C). However, stressinduced increases in plasma corticosterone levels did not differ between the IVA and SAL groups during exposure to the stressors (Fig. 1D, 2D, 3D).

\section{Discussion}

In our experiments, we have confirmed that ivabradine pretreatment significantly reduces heart rate during unstressed conditions. However, contrary to the generally accepted assumption that ivabradine does not affect blood pressure, we have shown that ivabradine administration even in dose of $5 \mathrm{mg} / \mathrm{kg}$ b.w. also significantly reduced baseline blood pressure in rats. Moreover, we found that ivabradine also attenuated stress-induced increases in heart rate and blood pressure. In rats exposed to handling (a mild stressor), ivabradine administration significantly reduced stressorinduced activation of SAS as shown by the reduced levels of EPI and NE in ivabradine-treated rats compared to salineinjected ones. However, handling-induced activation of the HPA axis, as determined by plasma corticosterone levels, was not affected by ivabradine. On the contrary, ivabradine significantly potentiated restraint- and immobilizationinduced increases of plasma EPI levels whereas plasma NE and corticosterone levels exhibited ambiguous changes.

To our knowledge, there is only one study in humans investigating the effect of ivabradine on plasma EPI and NE levels in healthy volunteers at basal conditions, as well as during tilt and physical activity. They found out that ivabradine administration was associated with decreased levels of heart rate and mean arterial pressure together with increased levels of EPI and NE during exercises with sympathetic stimulation (Joannides et al. 2006).

We hypothesize that the mechanisms responsible for reduced activation of SAS in rats pretreated with ivabradine and exposed to a mild stressor (handling) are related to mechanisms of learning by conditioning (for review see Ramsay and Woods 2016). Exposure of an organism to stressors usually leads to an increase in heart rate. Because activation of the neuroendocrine stress response and increases in heart rate are frequently coupled, it is possible that this response develops via conditioning. This conditioned response has the neuroendocrine stress response as the unconditioned factor and increases in heart rate as conditioned factor. In this way, an increase in heart rate is able to potentiate the neuroendocrine stress response by itself. The reduction of heart rate by ivabradine also reduces the effectiveness of this conditioned response and therefore may be responsible for the attenuated activity of SAS detected in handled rats pretreated by ivabradine.
In contrast to handling, plasma EPI was increased in rats pretreated by ivabradine and exposed to an intermediate stressor such as restraint stress, or a strong stressor such as immobilization. We hypothesize that this is because restraint stress and immobilization represent stressors that are accompanied by physical activity when animals try to escape from the restraint cylinder or immobilization board, as compared to handling, which does not. This physical activity may be accompanied by mild hypoxia in muscles that represents a potent stimulus for the adrenal medulla, whereas sympathetic nerves releasing NE are not significantly stimulated (Young et al. 1984).

In addition, we hypothesize that the ivabradine-induced reduction of heart rate in animals exposed to intermediate (restraint) or intensive (immobilization) stressors is associated with insufficient peripheral responses to released catecholamines into the bloodstream. For example, the ivabradine-induced decrease in heart rate and blood pressure may consequently lead to reduced perfusion of organs and muscles. This insufficient peripheral response to excessive catecholamine release provokes additional stimulation of SAS and subsequent EPI release as documented by the increased plasma EPI in ivabradine-injected rats exposed to restraint or immobilization. Published data indicate that activation of baroreflex may play a pivotal role in this compensatory response (Dias da Silva et al. 2015).

Ivabradine administration did not significantly affect stress-induced increases in plasma corticosterone levels. Therefore, we suggest that afferent signaling from the cardiovascular system plays a more important role in the regulation of SAS activity than the HPA axis. Furthermore, these data suggest that HPA axis activity is mainly under the influence of the forebrain structures activated predominantly by psychological stressors (Ziegler and Herman 2002; Keller-Wood 2015).

Our findings of an attenuated neuroendocrine stress response as a consequence of reduced heart rate during exposure to a mild stressor supports the assumption that while stress related processes in the brain, including emotions, drive changes in the activity of visceral organs, activation of visceral organs may in turn shape neuroendocrine stress response (Critchley and Harrison 2013). Because ivabradine does not cross blood-brain barrier (Young et al. 2014), its central effect on neuroendocrine stress response may be excluded. Based on our data, we suggest that the heart is activated during stress response by central commands originating in the brain, but the heart itself also modulates neuroendocrine and emotional responses in the brain, as proposed the James-Lange theory of emotions.

Transmission of signals from the heart to the brain is altered in patients who have received a heart transplant. However, published data indicate that the stress response is not affected in these patients (Salmon et al. 2001). This 
discrepancy between our findings and the above-mentioned data might be related to several factors. In our experiments, we selectively prevented the stress-induced increase in heart rate, while in patients with a transplanted heart, a mild stressor (Stroop test) induces a slight increase in heart rate and blood pressure that may lead to activation of several receptors in the cardiovascular system (e.g. mechanoreceptors in the heart, baroreceptors). In addition, re-innervation occurs in a transplanted heart. Moreover, these patients are treated by several drugs also affecting the heart itself. All of these factors may affect transmission of signals from the transplanted heart to the brain, consequently affecting the stress response and may explain the discrepancies between published data and our findings.

To our knowledge, this is the first study investigating the effect of attenuating stress-induced heart activity by ivabradine during the neuroendocrine stress response in laboratory animals exposed to different stressors. We have shown that attenuation of the stress-induced increase in heart rate during conditions of mild stress reduces the response of the SAS. These data indicate that afferent signals from the heart play a significant role in modulating the neuroendocrine stress response, which may highlight the importance of visceral signals in modulating emotions, as originally proposed by the James-Lange theory. Therefore, psychological approaches to reducing the stress-induced rise in heart rate may be of importance in neurotic individuals with an exaggerated response to stressors (Schneider et al. 2012). In addition, pharmacological attenuation of heart rate during stressful conditions may represent a protective strategy useful in preventing the adverse effects of exaggerated SAS activation, particularly in patients with cardiovascular diseases.

Acknowledgements. This research was supported by the VEGA grant (1/0300/15) and European Regional Development Fund Research and Development Grant (ITMS 26240120023). We wish to thank Dr. Ken Goldstein of ScienceDocs (www.sciencedocs.com) for the editing of this paper.

Conflict of interest. The authors declare that they have no conflicts of interest.

\section{References}

Bois P, Bescond J, Renaudon B, Lenfant J (1996): Mode of action of bradycardic agent, $S$ 16257, on ionic currents of rabbit sinoatrial node cells. Br. J. Pharmacol. 118, 1051-1057 https://doi.org/10.1111/j.1476-5381.1996.tb15505.x

Critchley HD, Harrison NA (2013): Visceral influences on brain and behavior. Neuron 77, 624-638 https://doi.org/10.1016/j.neuron.2013.02.008

Day HE, Nebel S, Sasse S, Campeau S (2005): Inhibition of the central extended amygdala by loud noise and restraint stress. Eur. J. Neurosci. 21, 441-454 https://doi.org/10.1111/j.1460-9568.2005.03865.x

Delahunt JW, Mellsop G (1987): Hormone changes in stress. Stress Med. 3, 123-134

https://doi.org/10.1002/smi.2460030209

Dias da Silva VJ, Tobaldini E, Rocchetti M, Wu MA, Malfatto G, Montano N, Zaza A (2015): Modulation of sympathetic activity and heart rate variability by ivabradine. Cardiovasc. Res. 108, 31-38 https://doi.org/10.1093/cvr/cvv180

DiFrancesco D, Camm JA (2004): Heart rate lowering by specific and selective I(f) current inhibition with ivabradine: a new therapeutic perspective in cardiovascular disease. Drugs 64, $1757-1765$ https://doi.org/10.2165/00003495-200464160-00003

DiFrancesco D (2006): Funny channels in the control of cardiac rhythm and mode of action of selective blockers. Pharmacol. Res. 53, 399-406 https://doi.org/10.1016/j.phrs.2006.03.006

Gardiner SM, Kemp PA, March JE, Bennett T (1995): Acute and chronic cardiac and regional haemodynamic effects of the novel bradycardic agent, S16257, in conscious rats. Br. J. Pharmacol. $115,579-586$ https://doi.org/10.1111/j.1476-5381.1995.tb14971.x

Goldstein DS (1995): Stress, Catecholamines, and Cardiovascular Disease. Oxford University Press Inc.; Oxford, New York

Guglin M (2013): Heart rate reduction in heart failure: ivabradine or beta blockers? Heart Fail. Rev. 18, 517-528 https://doi.org/10.1007/s10741-012-9347-6

Chrousos GP (2009): Stress and disorders of the stress system. Nat. Rev. Endocrinol. 5, 374-381 https://doi.org/10.1038/nrendo.2009.106

Jänig W (2006): The Integrative Action of the Autonomic Nervous System. Neurobiology of Homeostasis. Cambridge University Press, Cambridge https://doi.org/10.1017/CBO9780511541667

Joannides R, Moore N, Iacob M, Compagnon P, Lerebours G, Menard JF, Thuillez C (2006): Comparative effects of ivabradine, a selective heart rate-lowering agent, and propranolol on systemic and cardiac haemodynamics at rest and during exercise. Br. J. Clin. Pharmacol. 61, 127-137 https://doi.org/10.1111/j.1365-2125.2005.02544.x

Keller-Wood M (2015): Hypothalamic-pituitary--adrenal axisfeedback control. Compr. Physiol. 5, 1161-1182 https://doi.org/10.1002/cphy.c140065

Kvetnansky R, Mikulaj L (1970): Adrenal and urinary catecholamines in rats during adaptation to repeated immobilization stress. Endocrinology 87, 738-743

https://doi.org/10.1210/endo-87-4-738

Kvetnansky R, Silbergeld S, Weise VK, Kopin IJ (1971): Effects of restraint on rat adrenomedullary response to 2-deoxy-Dglucose. Psychopharmacologia 20, 22-31 https://doi.org/10.1007/BF00404055

Kvetnansky R, Sun C. L, Lake C. R, Thoa N, Torda T, Kopin IJ (1978): Effect of handling and forced immobilization on rat plasma levels of epinephrine, norepinephrine, and dopaminebeta-hydroxylase. Endocrinology 103, 1868-1874 https://doi.org/10.1210/endo-103-5-1868

Ling S, Jamali F (2003): Effect of cannulation surgery and restraint stress on the plasma corticosterone concentration in the rat: ap- 
plication of an improved corticosterone HPLC assay. J. Pharm. Pharm. Sci. 6, 246-251

Lipp MN, Anderson DE (1999): Cardiovascular reactivity to simulated social stress. Stress Med. 15, 249-257 https://doi.org/10.1002/(SICI)1099-1700(199910)15:4<249::AID-SMI821>3.0.CO;2-3

Masini CV, Nyhuis TJ, Sasse SK, Day HE, Campeau S (2011): Effects of voluntary wheel running on heart rate, body temperature, and locomotor activity in response to acute and repeated stressor exposures in rats. Stress 14, 324-334 https://doi.org/10.3109/10253890.2010.548013

McEwen BS, Gianaros PJ (2010): Central role of the brain in stress and adaptation: links to socioeconomic status, health, and disease. Ann. NY Acad. Sci. 1186, 190-222 https://doi.org/10.1111/j.1749-6632.2009.05331.x

Muntzel MS, Al-Naimi OA, Barclay A, Ajasin D (2012): Cafeteria diet increases fat mass and chronically elevates lumbar sympathetic nerve activity in rats. Hypertension 60, 1498-1502 https://doi.org/10.1161/HYPERTENSIONAHA.112.194886

Nawarskas JJ, Bowman BN, Anderson JR (2015): Ivabradine: a unique and intriguing medication for treating cardiovascular disease. Cardiol. Rev. 23, 201-211 https://doi.org/10.1097/CRD.0000000000000070

Peyronnet R, Nerbonne JM, Kohl P (2016): Cardiac mechano-gated ion channels and arrhythmias. Circ. Res. 118, 311-329 https://doi.org/10.1161/CIRCRESAHA.115.305043

Pozza RD, Kleinmann A, Bechtold S, Fuchs A, Netz H (2006): Reinnervation after heart transplantation in children: results of short-time heart rate variability testing. Pediatr. Transplant. 10, 429-433 https://doi.org/10.1111/j.1399-3046.2006.00485.x

Raczak G, La Rovere MT, Mortara A, Assandri J, Prpa A, Pinna GD, Maestri R, D'Armini AM, Vigano M, Cobelli F (1999): Arterial baroreflex modulation of heart rate in patients early after heart transplantation: lack of parasympathetic reinnervation. J. Heart. Lung Transplant. 18, 399-406 https://doi.org/10.1016/S1053-2498(98)00071-0

Ramsay DS, Woods SC (2016): Physiological regulation: how it really works. Cell Metab. 24, 361-364 https://doi.org/10.1016/j.cmet.2016.08.004
Salmon P, Stanford SC, Mikhail G, Zielinski S, Pepper JR (2001): Hemodynamic and emotional responses to a psychological stressor after cardiac transplantation. Psychosom. Med. 63, 289-299 https://doi.org/10.1097/00006842-200103000-00015

Schneider TR, Rench TA, Lyons JB, Riffle RR (2012): The influence of neuroticism, extraversion and openness on stress responses. Stress Health 28, 102-110 https://doi.org/10.1002/smi.1409

Thollon C, Cambarrat C, Vian J, Prost JF, Peglion JL, Vilaine JP (1994): Electrophysiological effects of S 16257, a novel sino-atrial node modulator, on rabbit and guinea-pig cardiac preparations: comparison with UL-FS 49. Br. J. Pharmacol. $112,37-42$ https://doi.org/10.1111/j.1476-5381.1994.tb13025.x

Thrivikraman KV, Huot RL, Plotsky PM (2002): Jugular vein catheterization for repeated blood sampling in the unrestrained conscious rat. Brain Research Brain Research Protocols 10, 84-94 https://doi.org/10.1016/S1385-299X(02)00185-X

Ulrich-Lai YM, Herman JP (2009): Neural regulation of endocrine and autonomic stress responses. Nat. Rev. Neurosci. 10, 397-409 https://doi.org/10.1038/nrn2647

Young GT, Emery EC, Mooney ER, Tsantoulas C, McNaughton PA (2014): Inflammatory and neuropathic pain are rapidly suppressed by peripheral block of hyperpolarisation-activated cyclic nucleotide-gated ion channels. Pain 155, 1708-1719 https://doi.org/10.1016/j.pain.2014.05.021

Young JB, Rosa RM, Landsberg L (1984): Dissociation of sympathetic nervous system and adrenal medullary responses. Am. J. Physiol. 247, E35-40 https://doi.org/10.1152/ajpendo.1984.247.1.E35

Ziegler DR, Herman JP (2002): Neurocircuitry of stress integration: anatomical pathways regulating the hypothalamo-pituitaryadrenocortical axis of the rat. Integr. Comp. Biol. 42, 541-551 https://doi.org/10.1093/icb/42.3.541

Received: September 5, 2018

Final version accepted: November 13, 2018

First published online: March 1, 2019 